\title{
Cell specific expression of peroxiredoxins in human lung and pulmonary sarcoidosis
}

\author{
V L Kinnula, S Lehtonen, R Kaarteenaho-Wiik, E Lakari, P Pääkkö, S W Kang, \\ S G Rhee, Y Soini
}

Thorax 2002;57: 157-164

See end of article for authors' affiliations

................

Correspondence to: Dr V Kinnula, University of Oulu, Department of Internal Medicine, Pulmonary Division, $\mathrm{P} O$ Box 5000 (Kajaanintie 50A), FIN-90014 Oulun Yliopisto, Finland; vuokko.kinnula@oulu.fi or vuokko.kinnula@ppshp.fi

Revised version received 17 September 2001 Accepted for publication 27 September 2001

\begin{abstract}
Background: Six proteins of the peroxiredoxin ( $P r x)$ family have recently been characterised which have the capacity to decompose hydrogen peroxide in vivo and in vitro. These proteins may have an important role in the protection of human lung against endogenous and exogenous oxidant stress. However, the expression and distribution of these proteins in healthy human lung and diseased lung tissue is unknown.

Methods: The cell specific expression of Prxs in healthy lung tissue from four non-smokers and in parenchymal tissue from 10 subjects with pulmonary sarcoidosis was investigated by immunohistochemistry, and expression of these proteins in various cultured lung cells and cells of bronchoalveolar lavage (BAL) fluid of controls and patients with sarcoidosis was assessed by Western blot analysis.

Results: All six Prxs could be synthesised in cultured human lung cells. The bronchial epithelium showed moderate to high expression of Prxs I, III, V and VI, the alveolar epithelium expressed mainly Prxs V and VI, and alveolar macrophages expressed mainly Prxs I and III. Granulomas of subjects with sarcoidosis expressed mainly Prxs I and III. Samples of BAL fluid from controls and from subjects with sarcoidosis had very similar findings, except that Prxs II and III had a tendency for increased immunoreactivity in sarcoidosis tissue.

Conclusions: Prxs I, III, V, and VI, in particular, have prominent and cell specific expression in human lung tissue. High expression of Prxs I and III in granulomas and alveolar macrophages of sarcoidosis parenchyma may have a significant effect on the oxidant burden and the progression of lung injury in this disease.
\end{abstract}

$\mathrm{T}$ he human lung presents unique tissue for the investigation of the effects of free radicals because lung cells are directly exposed to higher oxygen concentrations than most other tissues. Furthermore, lung cells have an increased oxidant burden during inflammation since a typical feature of many pulmonary disorders is the activation of inflammatory cells which leads to the respiratory burst and generation of reactive oxygen metabolites. Free radicals and antioxidants are also associated with multiple signal conducting pathways and activation or inhibition of transcription factors. In addition, the synthesis of growth factors and cytokines such as platelet derived growth factor (PDGF), epidermal growth factor (EGF), transforming growth factor beta $(\mathrm{TGF} \beta)$ and tumour necrosis factor alpha (TNF $\alpha$ ) may be influenced by free radicals. ${ }^{1}$ Antioxidant defence mechanisms can therefore be hypothesised to play a central role, not only in preventing oxidant associated lung diseases, but also in modulating the progression of these disorders.

The most important antioxidant enzymes (AOEs) scavenging superoxide include superoxide dismutases-namely, manganese superoxide dismutase (MnSOD), copper zinc superoxide dismutase (CuZnSOD), and extracellular superoxide dismutase (ECSOD). ${ }^{23}$ MnSOD is weakly expressed in alveolar macrophages and airway epithelial cells in particular and is induced by cytokines and inflammatory disorders of the lung, ${ }^{4-6}$ CuZnSOD is constitutively expressed in human bronchial and alveolar epithelium, ${ }^{67}$ and ECSOD occurs mainly in human alveolar macrophages, in areas containing high amounts of type I collagen fibres and near lung capillaries. ${ }^{8}$ The most widely investigated hydrogen peroxide scavenging mechanisms include catalase and glutathione dependent pathways such as glutathione peroxidase. ${ }^{3}$ Human bronchial epithelial cells, alveolar type II cells, and macrophages contain constitutive catalase and glutathione peroxidase reactivity, ${ }^{9-11}$ and the epithelial lining fluid of the human lung has a glutathione level more than 100 times higher than in circulating blood..$^{12}{ }^{13}$ In addition to these mechanisms, there are other hydrogen peroxide scavenging pathways which are poorly characterised but which may play a central role in the antioxidant defence of the human lung. Of these other mechanisms, cysteine containing small proteins such as thioredoxin (Trx) and peroxiredoxins (Prxs) are potentially of most interest. ${ }^{14-16}$ The Trx system has been described in human pulmonary sarcoidosis, ${ }^{17}$ but no studies have been conducted on any of the proteins of the Prx family in the human lung.

Peroxiredoxins belong to an antioxidant protein family which occurs in a wide variety of organisms from prokaryotes to mammals. The mammalian Prx group of proteins was originally divided into three subfamilies-Prxs I, II, and IIIall three having been detected in rat lung homogenates. ${ }^{18}$ Peroxiredoxin I is an inducible protein which is also found in some malignant tumours. ${ }^{19}$ Peroxiredoxins I and II are cytosolic while Prx III is synthesised in the cytosol and transported to the mitochondrial compartment of the cells. ${ }^{16}$ The mRNA of Prx IV has been detected in the homogenates of several tissues, including human lung, by Northern blotting analysis. ${ }^{20}$ It is mainly located in the endoplasmic reticulum but it functions also in the extracellular space and has heparin binding properties. ${ }^{21}$ Peroxiredoxin V, which shows $10 \%$ sequence identity to Prxs I-IV, was originally found in the bronchoalveolar lavage (BAL) fluid. ${ }^{22}{ }^{23}$ It is widely expressed in rat tissues and induced in rat lung homogenates at least by lipopolysaccharide. ${ }^{23}$ In addition to the cytosolic and peroxisomal localisation, Prx V was recently reported in the mitochondrial compartment in rat kidney cells. ${ }^{24}$ Interestingly, Prx V is located in the human chromosome 11 ql3 which is an important genetic region for atopic hypersensitivity. ${ }^{25} \mathrm{~A}$ recent study has reported a new mammalian cytosolic Prx (1-Cys 

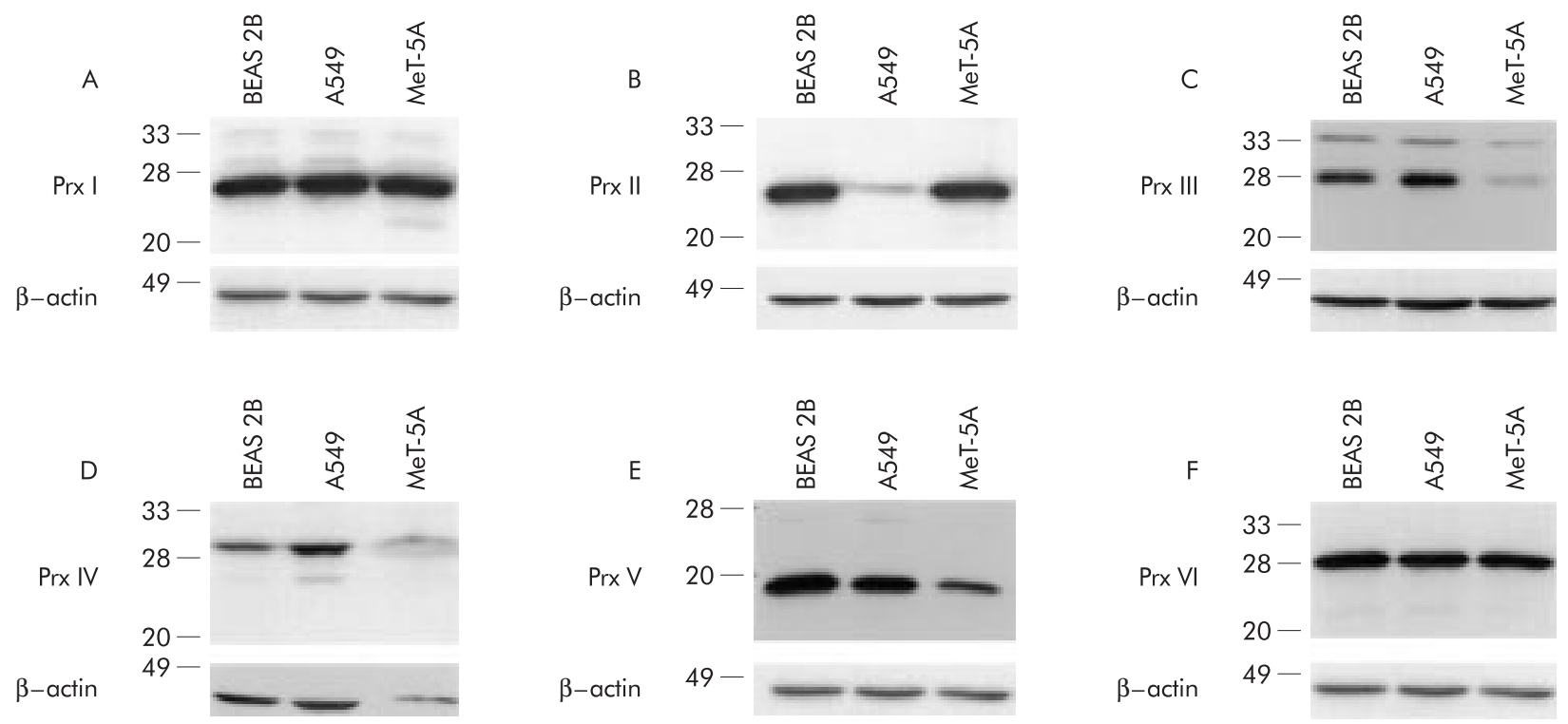

Figure 1 Expression of Prxs in various cultured human lung cell lines. Total cell extracts were prepared from BEAS 2B, A549, and MeT-5A cells. $75 \mu \mathrm{g}$ cell protein was separated by $12 \%$ SDS-PAGE. Immunoblotting was performed with (A) anti-Prx I, (B) anti-Prx II, (C) anti-Prx III, (D) anti-Prx IV, (E) anti-Prx V, and (F) anti-Prx VI antibodies. After detection the membranes were stripped of antibodies and redetected by

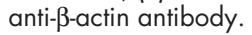

Prx) which contains, in contrast to the other Prxs, only one conserved cysteine ( $\mathrm{N}$-terminal cys 47$).{ }^{26}$ In the present study 1-Cys Prx has been named Prx VI. Although nearly all Prxs have been detected in lung homogenates, the distribution of these proteins has not previously been investigated in animal or human lung tissue. It can be hypothesised that these enzymes have individual distributions and expressions, they may be upregulated in oxidant related inflammatory lung diseases, and they may have an important role in protecting lung cells against oxidants.

To understand better the mechanisms by which human lung cells are protected against oxidants, we investigated the localisation and expression of all six Prxs in healthy human lung tissue by immunohistochemistry and in various lung cells and BAL fluid cells by Western blot analysis. To investigate the regulation of Prxs during inflammatory conditions in vivo their expressions were assessed in pulmonary sarcoidosis. Typical features of parenchymal sarcoidosis are widespread inflammation, formation of typical granulomas, and well preserved lung architecture.

\section{METHODS \\ Biopsies}

Lung biopsy specimens from four non-smoking subjects with normal histopathology undergoing surgery for carcinoid tumours and from 10 patients with parenchymal biopsy specimens consistent with sarcoidosis were retrieved from the files of the Department of Pathology, Oulu University Hospital by re-evaluating lung biopsy specimens taken either as open or thoracoscopic biopsies. All the sarcoidosis specimens were diagnostic; no anti-inflammatory treatment had been started in any case. The biopsy tissue was taken from different parts of the left or right lung and fixed in $10 \%$ formalin under vacuum to remove air bubbles or perfused by injecting the fixative using a small syringe into the bronchioles. ${ }^{27}$ Clinical data such as sex, age, and clinical diagnosis were obtained from hospital records.

\section{Bronchoalveolar lavage}

Fibreoptic bronchoscopy for sampling of BAL fluid was performed under local anaesthesia with lidocaine (lignocaine). Controls included subjects investigated for minor respiratory symptoms whose BAL fluid cell profile was normal. After centrifugation ( $400 \mathrm{~g}$ for 15 minutes) the cell pellets were stored at $-70^{\circ} \mathrm{C}$ for later analysis. Bronchoscopic examinations were conducted as differential diagnostic investigations. The study protocol had been accepted by the ethical committee of the hospital.

\section{Cell cultures}

Human non-malignant bronchial BEAS 2B cells which are SV40 transformed cells were obtained from the National Cancer Institute, Laboratory of Human Carcinogenesis (Dr Harris, Bethesda, MD, USA ${ }^{28}$ and cultured on uncoated petri dishes in serum free hormone supplemented medium according to the manufacturer's instructions (bronchial epithelial growth medium (BEGM), Clonetics Inc, San Diego, CA, USA). A549 alveolar epithelial cells, which also represent malignant lung adenocarcinoma cells, originating from type II pneumocytes (American Type Culture Collections) were maintained in Nutrient Mixture F-12 Ham (Gibco) growth medium supplemented with $15 \%$ heat inactivated fetal bovine serum (Gibco), $0.03 \%$ L-glutamine, $100 \mathrm{U} / \mathrm{ml}$ penicillin, and $100 \mu \mathrm{g} / \mathrm{ml}$ streptomycin (all from LTI, Life Technologies, Paisley, UK). Human MeT-5A mesothelial cells which are SV40 transformed cells (American Type Culture Collections) were grown in RPMI 1640 cell culture medium supplemented with $10 \%$ fetal calf serum.

\section{Histochemistry}

Rabbit anti-Prx antibodies have been described previously. ${ }^{16} 2023$ Immunostaining with the various Prxs was performed as follows. Before the application of the primary antibodies (dilutions 1:1500 for Prx I, 1:1000 for Prx II, 1:500 for Prx III, 1:1000 for Prx IV, 1:2000 for Prx V, and 1:2000 for Prx VI) $4 \mu \mathrm{m}$ sections of the biopsy specimens were dewaxed in xylene and rehydrated through a series of ethanol solutions, after which they were incubated in 3\% hydrogen peroxide for 15 minutes to eliminate endogenous peroxidase activity. Prior to hydrogen peroxide treatment the sections were incubated in $10 \mathrm{mM}$ citric acid monohydrate, $\mathrm{pH}$ 6.0, for 10 minutes and heated in a microwave oven, after which the sections were blocked by immersing them in $2 \%$ milk powder $\mathrm{dH}_{2} \mathrm{O}$ for 10 minutes. Immunostaining was performed using the Histostain-Plus Bulk kit (Zymed Laboratories Inc, South San 

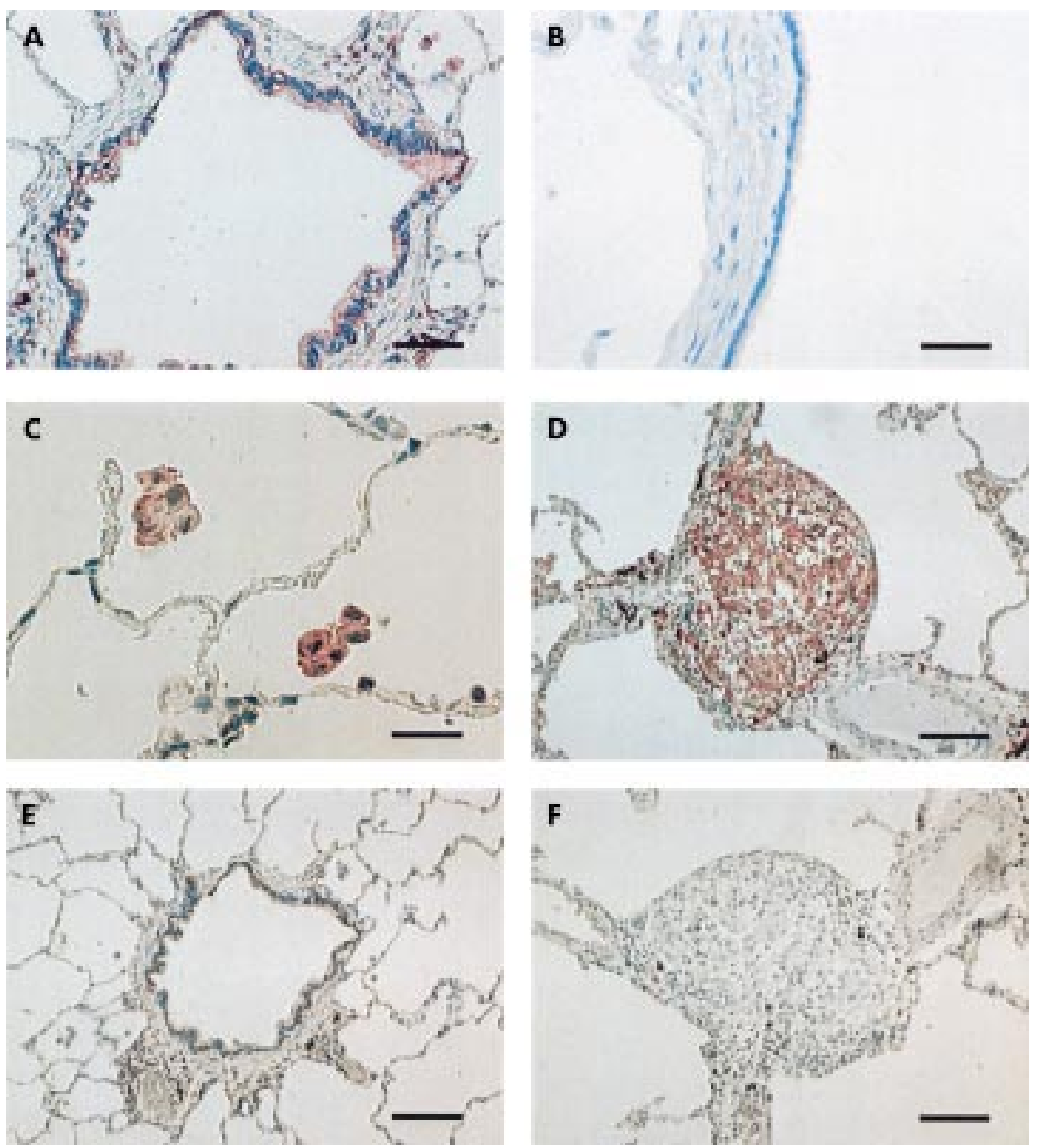

Figure 2 (A) Immunopositivity for Prx I in the cells of bronchial epithelium in the normal lung (scale bar 80 mm). (B) Serum isotype control with haematoxylin counterstain (scale bar 80 um). (C) Prx I positive alveolar macrophages in the normal lung (scale bar 40 um). (D) An immunopositive granuloma for $\operatorname{Prx} I$ in the lung of a patient with sarcoidosis (scale bar $80 \mu \mathrm{m}$ ). (E) Faint immunopositivity for Prx II in the bronchial epithelium of normal lung (scale bar 80 m). (F) A granuloma of a patient with sarcoidosis is nearly negative for Prx II (scale bar $80 \mu \mathrm{m})$.

Francisco, CA, USA) and the chromogen used was aminoethyl carbazole (AEC) (Zymed Laboratories Inc). Negative control stainings were carried out by substituting PBS and serum isotype controls (Zymed Laboratories Inc) for the primary antibodies.

The results of the immunostaining were evaluated semiquantitatively by dividing the staining reaction into four categories: $-=$ no immunostaining present; $+=$ weak cytoplasmic staining; $++=$ moderate cytoplasmic staining; $+++=$ strong cytoplasmic staining.

Immunoreactivity in alveolar macrophages, alveolar epithelial cells, bronchial epithelial cells, endothelial cells, and granulomas was assessed semiquantitatively by two investigators (YS, RKW). The two evaluations were compared using Cohen's kappa statistics and appeared to correlate well with each other $(\kappa=0.5818) .{ }^{29}$

\section{Western blotting}

The cell pellets were resuspended in sterile water, mixed with the electrophoresis sample buffer, and boiled for 5 minutes at $95^{\circ} \mathrm{C}$. The protein concentration of the samples was measured using the Bio-Rad protein assay (Bio-Rad Laboratories) and $75 \mu \mathrm{g}$ of cell protein was applied per lane to a $12 \%$ sodium dodecyl sulfate-polyacrylamide gel (SDS-PAGE). The gel was electrophoresed for 1.5 hours at $80 \mathrm{~V}$ and the protein transferred for $\mathrm{l} \mathrm{h}$ at $100 \mathrm{~V}$ onto Hybond enhanced chemiluminescence (ECL) nitrocellulose membranes (Amersham, Bucks, UK) in a Mini-Protean II Cell (Bio-Rad). The blotted membrane was incubated with rabbit antibody to Prxs I-VI (dilutions 1:1000) followed by treatment with an antibody conjugated to horseradish peroxidase diluted 1:5000-1:7000 (Jackson Immunoresearch Laboratories Inc). The same membranes were stripped of bound antibodies and detected for $\beta$-actin by using a monoclonal anti-actin antibody diluted 1:30 000 (Sigma, St Louis, Missouri, USA) followed by sheep anti-mouse antibody conjugated to horseradish peroxidase diluted 1:10000 (Amersham). The proteins were detected using the ECL system (Amersham) and the luminol excitation was imaged on $x$ ray film (Kodak, Rochester, NY, USA). The expressions were quantitated by scanning densitometry using a 300A computing densitometer and image quant software version 3.0 Fast Scan (Molecular Dynamics, Sunnyvale, CA, USA).

\section{RESULTS}

All six Prxs could be detected in cultured human bronchial epithelial cells, alveolar epithelial cells, and mesothelial cells (fig 1). The immunoreactivity of the Prxs is shown in figs 2, 3 , and 4 which indicate cell specific localisation for each Prx in tissue from both healthy lung and pulmonary sarcoidosis. 

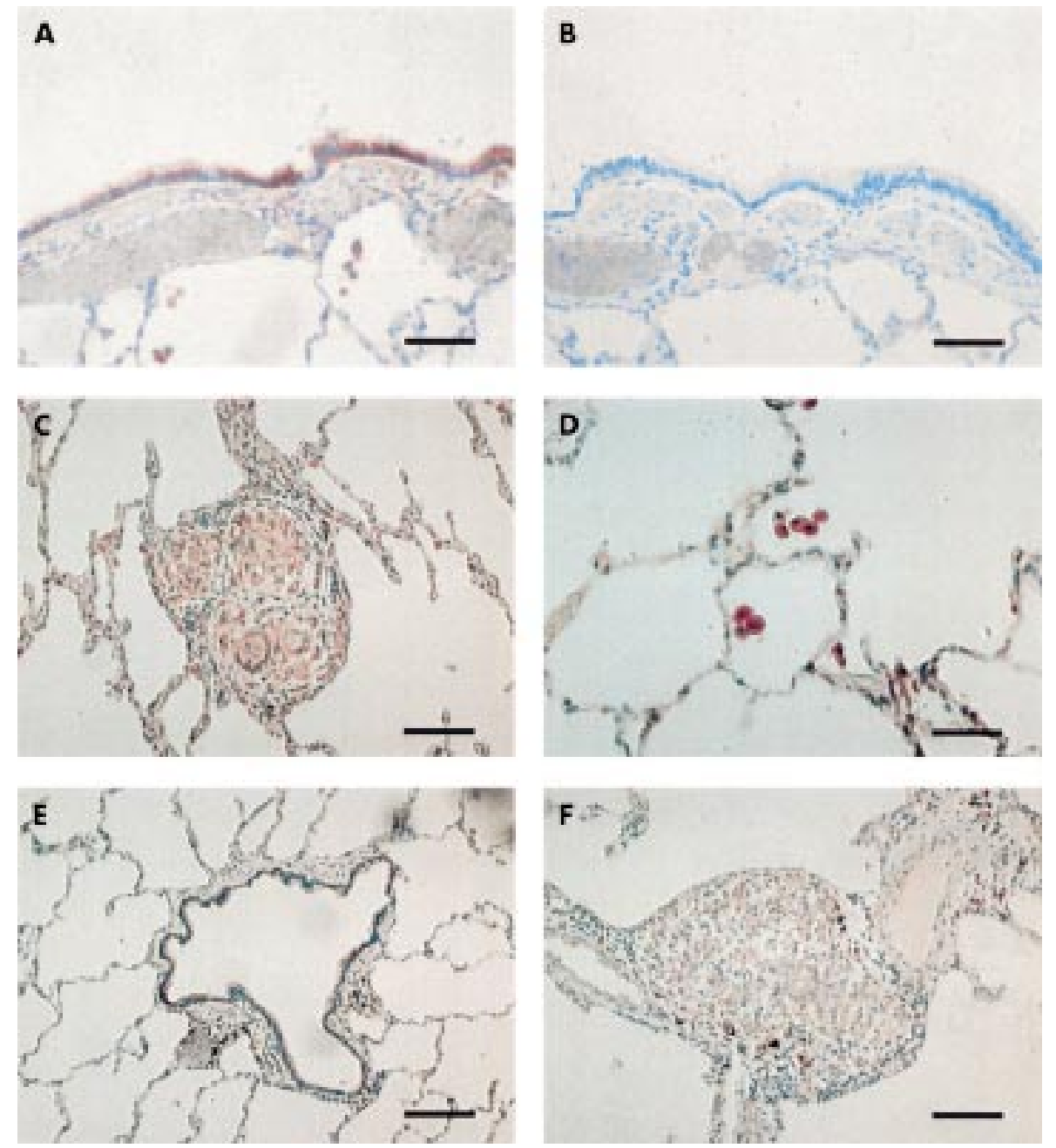

Figure 3 (A) Immunopositivity for Prx III in bronchial epithelial cells of the normal lung (scale bar $80 \mu \mathrm{m}$ ). (B) Serum isotype control with haematoxylin counterstain (scale bar $80 \mu \mathrm{m}$ ) in the same patient as in (A). (C) The giant cells of the sarcoid granuloma showing positivity for Prx III (scale bar $80 \mu \mathrm{m}$ ). (D) Prx III positive alveolar macrophages in normal lung (scale bar $80 \mu \mathrm{m}$ ). (E) Weak immunoreactivity for Prx IV in epithelial cells of the bronchiolus in normal lung (scale bar $80 \mu \mathrm{m})$. (F) Very weak positivity for Prx IV in granuloma of the lung in a patient with sarcoidosis (scale bar $80 \mu \mathrm{m}$ ).

Prx I was expressed especially in alveolar macrophages and bronchial epithelial cells, the intensities being at least moderate in three out of four cases in macrophages and in all four cases in the bronchial epithelium. Both alveolar epithelial cells and vascular endothelial cells showed generally weak positivity (3/4 cases; fig 2). Moderate to strong expression of Prx I was seen in the alveolar macrophages of sarcoidosis biopsy specimens $(8 / 10)$ and sarcoid granulomas (9/10), the immunoreactivity in alveolar pneumocytes of sarcoidosis also being at least moderate in six of the 10 cases (fig 2). Bronchial and alveolar epithelial cells in particular, and occasionally sarcoid granulomas and giant cells, also showed nuclear Prx I reactivity.

In contrast to Prx I, most biopsy specimens were only weakly or moderately positive for Prx II with none of the specimens showing strong reactivity. Bronchial epithelial cells had weak to moderate reactivity in all four cases, alveolar macrophages and epithelial cells being usually weakly positive (2/4). Plasma cells, lymphocytes, and occasionally endothelial cells were also weakly positive (fig 2 ). In sarcoidosis specimens macrophages showed weak immunoreactivity in five of the 10 cases; granulomas and giant cells were negative with the exception of two cases with weak reactivity (fig 2).

Prx III had a similar distribution to Prx I, with alveolar macrophages and bronchial epithelial cells showing intense reactivity in all four biopsy specimens and the alveolar epithe- lium showing moderate immunoreactivity (fig 3) in three of the four specimens. Prx III was also strongly positive in vascular endothelial cells. In sarcoidosis, alveolar macrophages and sarcoid granulomas were strongly positive in most of the cases (7/10; fig 3). As with Prx I, Prx III expression was also seen in fibroblasts in the fibrotic areas associated with sarcoid reactions. The cytoplasmic immunoreactivity was sometimes granular, reflecting the mitochondrial localisation of Prx III. ${ }^{1624}$ Occasional nuclear labelling is in agreement with the previously reported immunoreactivity of Prx III in isolated nuclear fractions. ${ }^{16}$

Prx IV reactivity was negative or weak in bronchial epithelium (2/4) and weak (3/4) in macrophages. Alveolar epithelial cells were negative except for occasional positivity observed in the metaplastic cells. Vascular endothelial cells were mostly negative. Alveolar macrophages of the patients with sarcoidosis and granulomas were weakly positive in seven of the 10 cases (fig 3).

Bronchial epithelium and alveolar type II cells revealed strong reactivity for Prx $\mathrm{V}$ in all four biopsy specimens, the reactivity varying from weak $(1 / 4)$ to strong $(1 / 4)$ in alveolar macrophages (fig 4). Bronchial epithelial cells and alveolar type II cells in the sarcoidosis biopsy specimens were strongly positive (10/10) whereas macrophages and sarcoid granulomas were mostly weakly positive (8/10 in both cases; fig 4$)$. 

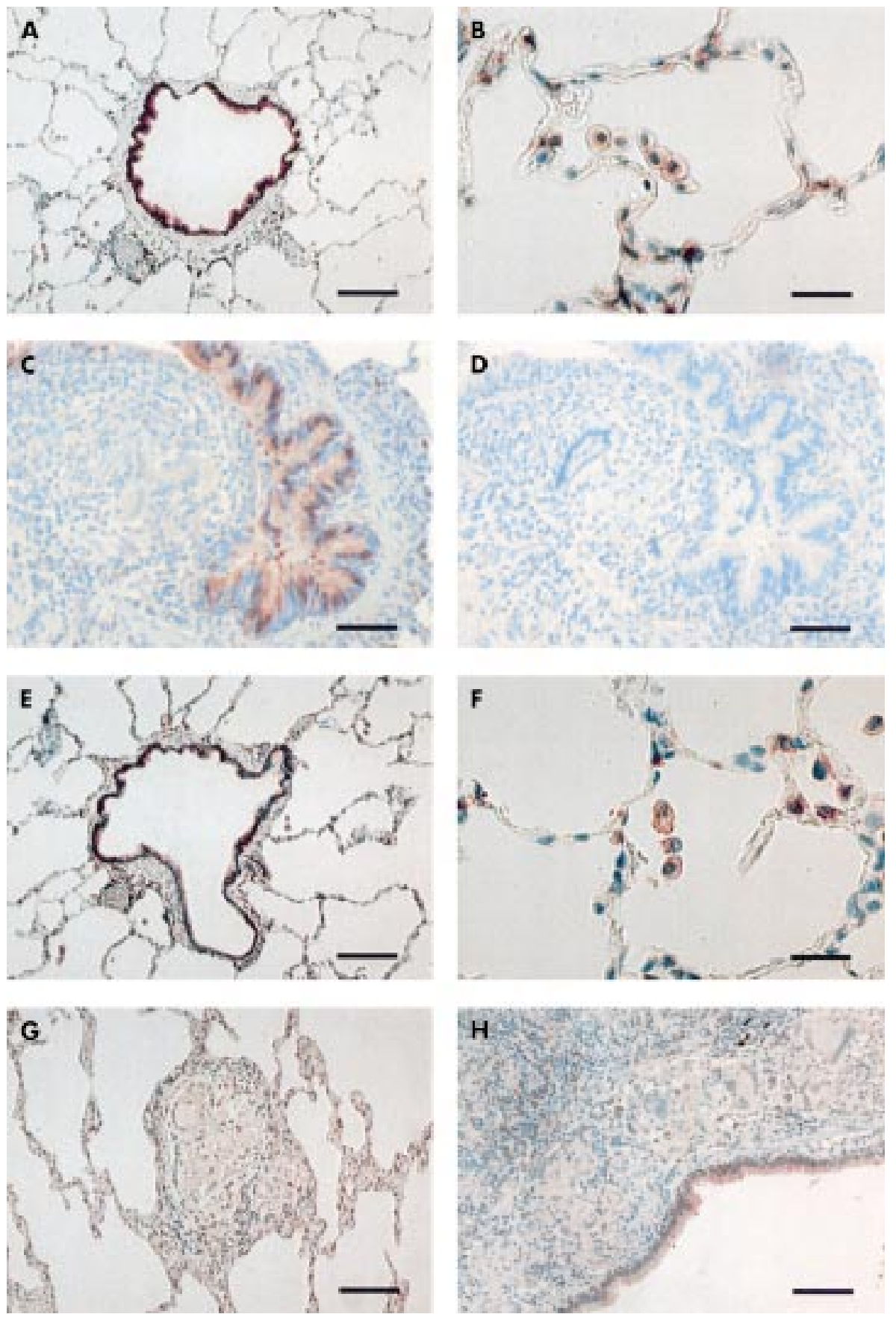

Figure 4 (A) Strong immunopositivity for $\operatorname{Prx} V$ in the epithelium of the bronchiolus in normal lung (scale bar 80 um). (B) Alveolar macrophages of normal lung showing positivity for $\operatorname{Prx} V$ (scale bar $40 \mu \mathrm{m})$. (C) Immunoreactivity for Prx V in the bronchial epithelial cells (on the right) and very weak immunoreactivity in the granuloma (on the left) in a patient with sarcoidosis (scale bar 40 um). (D) Serum isotype control with haematoxylin counterstain in the same patient as in (C) (scale bar $40 \mu \mathrm{m})$. (E) Positive Prx VI in the epithelium of the bronchiolus in normal lung (scale bar $80 \mu \mathrm{m}$ ). (F) Prx VI positive alveolar macrophages in normal lung (scale bar $40 \mu \mathrm{m}$ ). (G) Immunoreactivity for Prx VI in the granuloma of a patient with sarcoidosis (scale bar $80 \mu \mathrm{m})$. (H) Immunoreactivity for Prx VI in the giant cells of a sarcoid granuloma underlying the bronchial epithelium which is clearly positive for $\operatorname{Prx} \mathrm{VI}$ (scale bar $40 \mu \mathrm{m}$ ).

Positive labelling could be detected in the nuclear and cytosolic compartments of the cells. The cytoplasmic reaction was granular, which is consistent with the localisation of Prx $\mathrm{V}$ in the mitochondria. ${ }^{16}$

Prx VI expression was very similar to Prx V, showing most prominent immunoreactivity in the bronchial epithelium and alveolar type II cells with three of the four cases being strongly positive (fig 4). Sarcoid granulomas displayed at least moderate positivity in eight of the 10 cases and alveolar macrophages in five (fig 4). Nuclear labelling was frequently found, especially in bronchial and alveolar epithelial type II cells, which is consistent with recent data in nuclear fractions. ${ }^{26}$

A summary of the distribution and expression of Prxs in control lung biopsy specimens is shown in table 1 and fig 5 shows their expression in specimens from patients with pulmonary sarcoidosis.

Western blotting showed very similar expression of all Prxs in the BAL fluid samples of the controls and patients with sarcoidosis. When the immunoreactivities were standardised against $\beta$-actin, the relative expressions of various Prxs in the 
Table 1 Cell specific distribution and expression of various peroxiredoxins (Prxs) in normal human lung

\begin{tabular}{|c|c|c|c|c|c|}
\hline Protein & $M W(k D a)$ & Bronchial epithelial cells & Alveolar epithelial cells & Alveolar macrophages & Vascular endothelial cells \\
\hline $\operatorname{Prx} 1$ & 22 & ++ & + & ++ & + \\
\hline $\operatorname{Prx} \|$ & 22 & + & + & + & - \\
\hline Prx III & 28 & +++ & ++ & +++ & + \\
\hline $\operatorname{Prx} I V$ & 31 & + & - & + & - \\
\hline Prx V & 17 & +++ & +++ & ++ & - \\
\hline $\operatorname{Prx} \mathrm{VI}$ & 25 & +++ & +++ & ++ & + \\
\hline
\end{tabular}

Values are means from four separate tissue biopsy specimens obtained from control subjects. Intensities are shown as weak $(+)$ to strong $(+++)$ as described in the Methods section. The difference between the published molecular weights of Prx III and VI (fig 1 and table 1) is due to post-translational modifications as published earlier. ${ }^{16202326}$
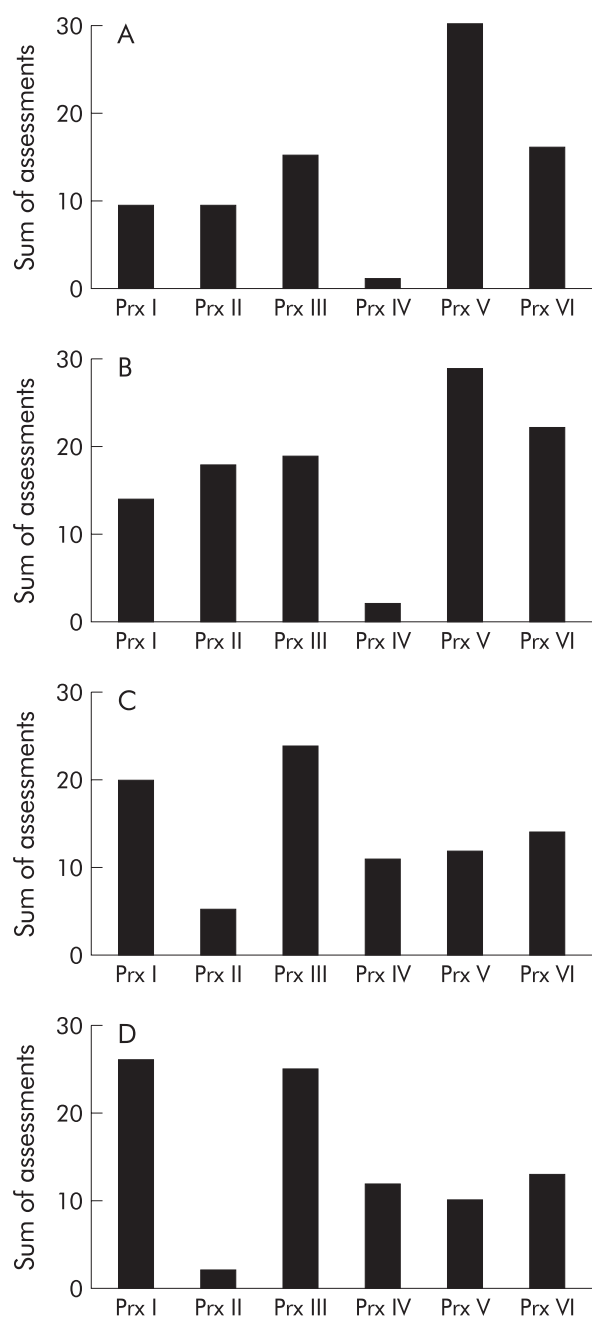

Figure 5 Distinct Prxs have different patterns of expression in pulmonary sarcoidosis. The sums of the assessments were derived from the reactivities in histological samples as described in the text in (A) bronchial epithelial cells, (B) alveolar epithelial cells, (C) alveolar macrophages, and (D) sarcoid granulomas.

BAL fluid of sarcoidosis tissue compared with control biopsy specimens were as follows: Prx I $-20 \%$, Prx II $+62 \%$, Prx III $+18 \%$, Prx IV -6\%, Prx V - 13\%, and Prx VI -17\% (fig 6).

\section{DISCUSSION}

This study shows for the first time that, in addition to the classical hydrogen peroxide scavenging pathways, catalase and glutathione associated mechanisms, human lung cells express all six members of the Prx family in a cell specific manner and that these proteins may also be induced in the granulomas of pulmonary sarcoidosis.
Hydrogen peroxide scavenging pathways may have an important antioxidant role in human airway epithelium. Previous studies on other hydrogen peroxide scavenging antioxidant mechanisms have shown that human bronchial epithelial cells express constitutive catalase reactivity ${ }^{79}{ }^{10}$ and that the mRNA of gamma-glutamyl cysteine synthetase ( $\gamma \mathrm{GCS})$ is upregulated at least by cigarette smoke in the airway epithelial cells of human lung. ${ }^{30}$ On the other hand, the specific activity of catalase in human bronchial epithelial cells has been suggested to be low. ${ }^{9}$ Further, neither catalase nor extracellular glutathione peroxidase are induced during acute oxidant stress in human bronchial epithelial cells. ${ }^{911}$ Additionally, only part of the hydrogen peroxide scavenging capacity of cultured human bronchial epithelial cells can be inhibited by aminotriazole (an inhibitor of catalase) or BSO (an inhibitor of $\gamma \mathrm{GCS}$, rate limiting enzyme in glutathione synthesis). ${ }^{7}$ These previous results may be partly explained by Prxs, since the bronchial epithelium of histopathologically normal lung revealed at least moderate or high expression of Prxs III, V, and VI in most of the cases. Our unpublished studies have also shown that human bronchial epithelium contains Trx and Trx reductase, and that Trx expression is enhanced in the bronchial epithelial cells of patients with different types of interstitial pneumonias. The interpretation of the relative importance of Prxs, Trxs, and the classical hydrogen peroxide scavenging AOEs in human airways remains unclear. The relatively high expression of several Prxs in normal bronchial and alveolar epithelium and alveolar macrophages suggests that these proteins constitute an important primary defence system in human lung.

Alveolar epithelium, especially type II pneumocytes, are oxidant resistant cells in vitro and in vivo. ${ }^{31}{ }^{32}$ Type II pneumocyte cells express high levels of catalase constitutively and they scavenge exogenous hydrogen peroxide mainly by a catalase dependent mechanism in vitro. ${ }^{1033}$ On the other hand, because of the high $\mathrm{Km}$ of catalase towards hydrogen peroxide, other mechanisms are probably needed to protect the alveolar epithelium during persistent oxidant stress in vivo. The findings of the present study suggest that Prxs V and VI, at least, may explain part of this resistance. Given that alveolar and bronchial epithelial cells change in culture, ${ }^{73}$ cultured cells cannot be directly used in the assessment of the antioxidant defence of the lung. We have shown that cultured alveolar epithelial cells, as well as bronchial epithelial cells and pleural mesothelial cells, express all members of the Prx family, so these cells may also be used to investigate the regulation of Prxs in human lung.

Alveolar macrophages are more resistant than neutrophils and have a longer half life; both of these factors may be partly associated with intracellular antioxidant defence. Human macrophages and monocytes contain multiple antioxidant mechanisms; they have a higher glutathione content than neutrophils ${ }^{34}$ and they scavenge hydrogen peroxide mainly by a catalase dependent pathway, but again part of the hydrogen peroxide consumption may be associated with pathways other than catalase or glutathione reductase. ${ }^{35}{ }^{36}$ We found that Prxs I, III, and V were at least moderately expressed in alveolar 

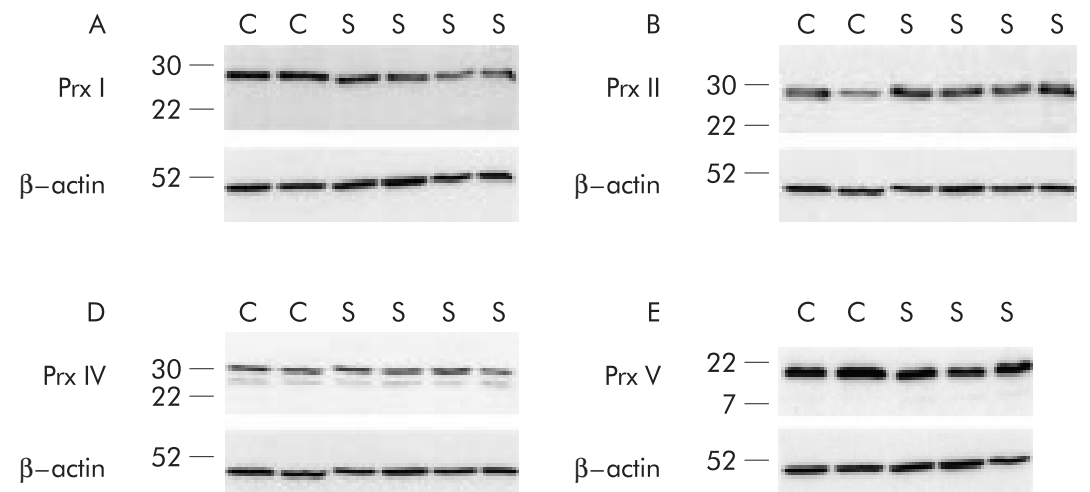

$\beta$-actin

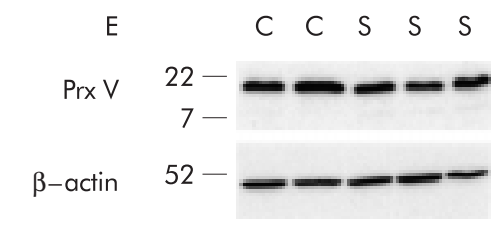

Figure 6 Western blot analysis of BAL fluid cells with anti-Prx antibodies. BAL fluid cells were obtained from two controls (C) and four patients with sarcoidosis (S). Total cell extracts were prepared and analysed as in fig 1 .

macrophages in most cases, suggesting their importance in protecting alveolar macrophages against oxidant stress.

The expression of Prxs I, III, V, and VI in macrophages and/or type II pneumocytes of control lung biopsy specimens suggests that these enzymes have constitutive activity in the human lung. On the other hand, at least some of these proteins may be induced by oxidants and cytokines mediated by transcription factors such as NF- $\mathrm{\kappa B} .{ }^{16}$ The biopsy specimens were taken from non-smokers with normal lung histopathology. However, all subjects had been anaesthetised and ventilated with elevated oxygen tension, so induction of these enzymes cannot be completely excluded. Induction of Prxs may theoretically also occur during bronchoscopy, so caution is required in interpreting the levels of various Prxs in healthy human lung tissue and BAL fluid. Furthermore, the activity of Prxs is regulated both at the expressional and protein levels. ${ }^{20}$ Prxs are capable of forming heterodimers and homodimers, both of which also modulate their activity. ${ }^{20}$

We used sarcoidosis as a model to investigate the regulation of Prxs in human lung tissue in vivo since the parenchyma in pulmonary sarcoidosis contains widespread inflammation, typical granulomas, but well preserved lung structure with a good prognosis. Previous studies have shown that one of the most important AOEs, MnSOD, which is induced by inflammatory cytokines, ${ }^{4}$ is increased in the macrophages and granulomas of pulmonary sarcoidosis. ${ }^{6}$ A recent study also showed induction of Trx in the BAL fluid of patients with sarcoidosis. ${ }^{17}$ In addition to MnSOD and Trx, high reactivities of Prxs I and III in granulomas and alveolar macrophages from patients with sarcoidosis, together with the possible induction of Prx II and III in the cells of the BAL fluid, suggest that many antioxidant mechanisms contribute to the protection of human lung against the oxidant stress in granulomatous lung diseases. It remains unclear whether induction or downregulation of these proteins plays a significant role in the progression of these lung disorders.

In conclusion, human lung cells express all six Prxs so far reported in a cell specific manner. The expression of several members of the Prx family was very similar in control lung tissue and parenchymal tissue of sarcoidosis, suggesting the importance of Prxs in the primary oxidant resistance of human lung. High expression of Prxs I and III in granulomas and alveolar macrophages and Prx $\mathrm{V}$ in the bronchial and alveolar epithelium of patients with sarcoidosis may also have important effects against the progression of this disease. Further studies will be necessary to evaluate the relative importance of various antioxidant mechanisms and these newly characterised proteins in human lung and lung diseases.

\section{ACKNOWLEDGEMENTS}

The authors acknowledge the technical assistance of Manu Tuovinen and Raija Sirviö. This work has been partly supported by the Finnish
Antituberculosis Association Foundation, Juselius Foundation and Cancer Society of Finland.

\section{Authors' affiliations}

V L Kinnula, S Lehtonen, R Kaarteenaho-Wiik, E Lakari, P Pääkkö, Y Soini, Departments of Internal Medicine and Pathology, University of Oulu and Oulu University Hospital, Finland

S W Kang, S G Rhee, Laboratory of Cell Signalling, National Institutes of Health, Bethesda, Maryland 20892, USA

\section{REFERENCES}

1 Thannickal VJ, Fanburg BL. Reactive oxygen species in cell signalling Am J Physiol 2000;279:L1005-28.

2 Fridovich I. Superoxide radical and superoxide dismutases. Ann Rev Biochem 1995;65:97-112.

3 Halliwell B, Gutteridge JM. Free radicals in biology and medicine. New York: Oxford University Press, 1989.

4 Wong GH, Goeddel DV. Induction of manganous superoxide dismutase by tumor necrosis factor, possible protective mechanism. Science 1988;242:941-4.

5 Coursin DB, Cihla HP, Oberley TD, et al. Immunolocalization of antioxidant enzymes and isozymes of glutathione S-transferase in normal rat lung. Am J Physiol 1992;263:L679-91.

6 Lakari E, Pääkkö P, Kinnula VL. Manganese superoxide dismutase, but not CuZn superoxide dismutase, is highly expressed in the granulomas of pulmonary sarcoidosis and extrinsic allergic alveolitis. Am J Respir Crit Care Med 1998;158:589-96.

7 Kinnula VL, Yankaskas JR, Chang L, et al. Primary and immortalized (BEAS 2B) human bronchial epithelial cells have significant antioxidative capacity in vitro. Am J Respir Cell Mol Biol 1994;11:568-76.

8 Oury TD, Chang LY, Marklund SL, et al. Immunocytochemical localization of extracellular superoxide dismutase in human lung. Lab Invest 1994;70:889-98.

9 Erzurum SC, Danel C, Gillissen A, et al. In vivo antioxidant gene in human airway epithelium for normal individuals exposed to $100 \% \mathrm{O}_{2}$. J Appl Physiol 1993;75: 1256-62.

10 Lakari E, Pääkkö P, Pietarinen-Runtti $P$, et al. Manganese superoxide dismutase and catalase are coordinately expressed in the alveolar region in chronic interstitial pneumonia and granulomatous diseases of the lung. Am J Respir Crit Care Med 2000;161:615-21.

11 Comhair SA, Thomassen M, Erzurum SC. Differential induction of extracellular glutathione peroxidase and nitric oxide synthase 2 in airway of healthy individuals exposed to $100 \% \mathrm{O}_{2}$ or cigarette smoke. Am J Respir Cell Mol Biol 2000;23:350-4.

12 Cantin AM, North SL, Hubbard RC, et al. Normal alveolar epithelial lining fluid contains high levels of glutathion. J Appl Physiol 1987;63:152-7.

13 Rahman I, MacNee W. Regulation of redox glutathione levels and gene transcription in lung inflammation: therapeutic approaches. Free Radic Biol Med 2000;28: 1405-20.

14 Powis G, Mustacich D, Coon A. The role of the redox protein thioredoxin in cell and cancer. Free Radic Biol Med 2000;29:312-22.

15 Holmgren $\mathbf{A}$. Redox regulation by thioredoxin and thioredoxin reductase. Biofactors 2000;11:63-4.

16 Kang SW, Chae HZ, Seo MS, et al. Mammalian peroxiredoxin isoforms can reduce hydrogen peroxide generated in response to growth factors and tumor necrosis factor- $\alpha$. J Biol Chem 1998;273:6297-302.

17 Koura T, Gon Y, Hashimoto S, et al. Expression of thioredoxin in granulomas of sarcoidosis: possible role in the development of $\mathrm{T}$ lymphocyte. Thorax 2000;55:755-61.

18 Chae HZ, Kim HJ, Kang SW, et al. Characterization of three isoforms of mammalian peroxiredoxin peroxides in the presence of thioredoxin. Diabetes Res Clin Pract 1999;45:101-12. 
19 Yanagawa T, Iwasa S, Ishii $\mathrm{T}$, et al. Peroxiredoxin I expression in oral cancer: a potential new tumor marker. Cancer Lett 2000;156:27-35.

20 Jin DY, Chae HZ, Rhee SG, et al. Regulatory role for a novel human thioredoxin peroxidase in NF-KB activation. J Biol Chem 1997:272:30952-61.

21 Okado-Matsumoto A, Matsumoto A, Fujii J, et al. Peroxiredoxin IV is a secretable protein with heparin-binding properties under reduced conditions. J Biochem 2000;1 27:493-501.

22 Knoops B, Clippe A, Bogard C, et al. Cloning and characterization of AOEB 166, a novel mammalian antioxidant enzyme of the peroxiredoxin family. J Biol Chem 1999:274:3051-8.

23 Seo MS, Kang SW, Kim K, et al. Identificaton of a new type of mammalian peroxiredoxin that forms an intramolecular disulfide as a reaction inermediate. J Biol Chem 2000;275:20346-54

24 Oberley TD, Verwiebe E, Zhong W, et al. Localization of the thioredoxin system in normal rat kidney. Free Radic Biol Med 2001;30:412-24.

25 Hemmann S, Blaser K, Crameri R. Allergens of Aspergillus fumigatus and Candida boidinii share IgE-binding epitopes. Am J Respir Crit Care Med 1997;156:1956-62.

26 Kang SW, Baines IC, Rhee SG. Characterization of a mammalian peroxiredoxin that contains one conserved cysteine. J Biol Chem 1998;273:6303-11

27 Dail DH, Hammar P. Pulmonary pathology. 2nd ed. New York: Springer-Verlag, 1994: 18-19, 597-678, 751-8.

28 Reddel RR, Ke Y, Gerwin BI, et al. Transformation of human bronchial epithelial cells by infecting with SV4O or adenovirus-12 SV4O hybrid virus and transfection via strontium phosphate coprecipitation with a plasmid containing SV40 early egion genes. Cancer 1988;48:1904-9.

29 Silcocks PBS. Measuring repeatability and validity of histological diagnosis: a brief review with some practical axamples. J Clin Pathol 1983:36: 1269-75

30 Rahman I, van Schadewijk AA, Hiemstra PS, et al. Localization of gamma-glutamylcysteine synthetase messenger RNA expression in lungs of smokers and patients with chronic obstructive pulmonary disease. Free Radic Biol Med 2000;28:920-5.

31 Crapo JD, Peters-Golden M, Marsh-Salin J, et al. Pathologic changes in the lungs of oxygen-adapted rats: a morphometric analysis. Lab Invest 1978;39:640-53.

32 Kinnula VL, Crapo JD, Raivio KO. Generation and disposal of reactive oxygen metabolites in the lung. Lab Invest 1995;73:3-19.

33 Kinnula VL, Chang L, Everitt Jl, et al. Oxidants and antioxidants in alveolar epithelial type II cells: in situ, freshly and cultured cells. Am J Physiol 1992;262:L69-77.

34 Pietarinen-Runtti $\mathbf{P}$, Lakari E, Raivio KO, et al. Expression of antioxidant enzymes in human inflammatory cells. Am J Physiol 2000;278: C 1 18-25.

35 McDonald RJ, Berger EM, Repine JE. Alveolar macrophage antioxidants prevent hydrogen peroxide mediated lung damage. Am Rev Respir Dis 1991;143:1088-91.

36 Pietarinen P, Raivio K, Devlin RB, et al. Catalase and glutathione reductase protection of human alveolar macrophages during oxidant exposure in vitro. Am J Respir Cell Mol Biol 1995;13:434-41. 